\begin{tabular}{|c|l|}
\hline Title & Observing Quantum Correlation of Photons in Laguerre Gauss Modes Using the Gouy Phase \\
\hline Author(s) & Kawase, Dai suke; Miyamoto, Y oko; Takeda, Mitsuo; Sasaki, Keiji; Takeuchi, Shigeki \\
\hline Citation & $\begin{array}{l}\text { Physical Review Letters, 101(5), 050501 } \\
\text { https://doi.org/L0.1103/PhysRevLett.101.050501 }\end{array}$ \\
\hline Issue Date & 2008-08-01 \\
\hline Doc URL & http://hdl.handle.net/2115/49846 \\
\hline Rights & ○ 2008 The American Physical Society \\
\hline Type & article \\
\hline File Information & PRL101-5_050501.pdf \\
\hline
\end{tabular}

Instructions for use 


\title{
Observing Quantum Correlation of Photons in Laguerre-Gauss Modes Using the Gouy Phase
}

\author{
Daisuke Kawase, ${ }^{1}$ Yoko Miyamoto, ${ }^{2}$ Mitsuo Takeda, ${ }^{2}$ Keiji Sasaki, ${ }^{1}$ and Shigeki Takeuchi ${ }^{1,3, *}$ \\ ${ }^{1}$ Research Institute for Electronic Science, Hokkaido University, Sapporo 060-0812, Japan \\ ${ }^{2}$ Department of Information and Communication Engineering, The University of Electro-Communications, Chofu 182-8585, Japan \\ ${ }^{3}$ The Institute of Scientific and Industrial Research, Osaka University, Ibaraki, Osaka 567-0047, Japan
}

(Received 11 March 2008; published 29 July 2008)

\begin{abstract}
The effect of the Gouy phase, which is one of the geometrical phases of photons, is observed through quantum correlation in Laguerre-Gaussian (LG) modes. In an experiment, the relative phase of two different LG modes of measurement basis states is manipulated via the Gouy phase, and the observed coincidence count rates agree well with theoretical predictions. This result suggests that the Gouy phase can be used as a new tool to manipulate multidimensional photonic quantum states.
\end{abstract}

DOI: 10.1103/PhysRevLett.101.050501

Manipulation of photonic quantum bits, or qubits, is critical in many important applications in quantum information processing, including quantum key distribution [1], quantum computing [2], dense coding [3], and teleportation [4]. Furthermore, interest in multidimensional photonic quantum states, or qunits, has grown recently because of the potential they have to realize new types of quantum communication protocols [5-7]. For realizing multidimensional states, photons in Laguerre-Gaussian (LG) modes have been attracting a lot of attention recently [8-12]. LG modes form an orthogonal basis set of paraxial solutions to the wave equation and have screw phase dislocations $\exp (i l \varphi)$, where $l$ is referred to as the azimuthal mode index. Photons in LG modes have orbital angular momentum $l \hbar[13,14]$.

Gouy phase shift is another interesting characteristic of photons in LG modes $[15,16]$. The Gouy phase shift is the axial phase shift that converging photons experience as they pass through the waist of the beam, and it is proportional to the azimuthal mode index $l$. In a paper [17], the Gouy phase shift is introduced as a manifestation of general Berry's phase [18]. Recently, Gouy phase shifts have been observed directly [19-22] or using an inteferometric technique [23]. However, neither the effect of Gouy phase for entangled state nor the manipulation of photonic quantum states using Gouy phase has been reported.

In this Letter, we propose a method for manipulating the quantum state of photons in LG modes using the Gouy phase shift and apply this method to observe the quantum correlation in LG modes of photons. In our method, the Gouy phase shift of LG modes is controlled by translating the beam waist position. Since the Gouy phase shift is proportional to the azimuthal mode index $l$ of the phase dislocation, it is in principle possible to manipulate the relative phases between more than two LG modes (i.e., $l=$ $0,1,2, \ldots)$ simultaneously.

Furthermore, we experimentally confirm the manipulation of Gouy phase shift through the entanglement of two photons generated via spontaneous parametric down conversion (SPDC). One of the two photons entangled in LG
PACS numbers: 03.67.Bg, 42.50.Ex, 42.50.Tx, 42.65.Lm

modes is detected as a superposition state between two different LG modes ( $l=0$ and 1$)$ with a Gouy phase shift, while the amplitudes and phases of the measurement basis state of the other photon are scanned two-dimensionally using the conventional method [24]. From the twodimensional (2D) map of the coincidence count rates, the observed phase shift agrees well with that predicted by theory. The direct observation of the Gouy phase shift, which is one of the geometric phases of photons, through quantum entanglement is interesting both in terms of fundamental physics and new technological applications.

The normalized LG mode with a beam waist located at $z=z_{0}$ is given in cylindrical coordinates by

$$
\begin{aligned}
\mathrm{LG}_{p l}\left(\rho, \varphi, z ; \omega_{0}, z_{0}\right)= & \sqrt{\frac{2 p !}{\pi(|l|+p) !}} \frac{1}{\omega}\left(\frac{\sqrt{2} \rho}{\omega}\right)^{|l|} L_{p}^{|l|}\left(\frac{2 \rho^{2}}{\omega^{2}}\right) \\
& \times \exp \left\{-\rho^{2}\left(\frac{1}{\omega^{2}}-\frac{i k}{2 R}\right)+i l \varphi-i \Psi_{p l}\right\},
\end{aligned}
$$

where $(\rho, \varphi, z)$ denote cylindrical coordinates, $k$ is the wave number, and $L_{p}^{|l|}(x)$ is the generalized Laguerre polynomial. $l$ is the azimuthal mode index, and $p$ is the radial mode index, which is related to the number of radial nodes. The parameters $R(z), \omega(z)$, and $\Psi_{p l}(z)$ denote the radius curvature of wave fronts, the beam radius, and the Gouy phase at the propagation distance $z$, respectively: $\omega(z)=$ $\omega_{0} \sqrt{1+\left[\left(z-z_{0}\right) / z_{R}\right]^{2}}, \quad R(z)=z\left\{1+\left[z_{R} /\left(z-z_{0}\right)\right]^{2}\right\}$, $\Psi_{p l}(z)=(2 p+|l|+1) \arctan \left[\left(z-z_{0}\right) / z_{R}\right] \quad$ with the Rayleigh range: $z_{R}=k \omega_{0}^{2} / 2$, where $\omega_{0}$ is the radius of the beam waist.

A photon state in $\mathrm{LG}_{p l}$ mode with a beam waist at $z=z_{0}$ that has a radius of $\omega_{0}$ is given by $[25,26]$

$$
\left|p l\left(\omega_{0}, z_{0}\right)\right\rangle=\int d r_{\perp} \mathrm{LG}_{p l}\left(r_{\perp}, z ; \omega_{0}, z_{0}\right) a^{\dagger}\left(r_{\perp}, z\right)|0\rangle,
$$

where $r_{\perp}=(\rho, \varphi),|0\rangle$ is the vacuum state and $a^{\dagger}\left(r_{\perp}, z\right)$ is the creation operator of a single photon at position $\left(r_{\perp}, z\right)$. 
Equation (2) implies that the phase of the photonic state in a certain $\mathrm{LG}_{p l}$ mode depends on the position of the beam waist, mainly due to the Gouy phase shift $\Psi_{p l}$ in Eq. (1).

Next, we explain how the relative phase between different LG modes can be controlled using Gouy phase. Figure 1 shows a plot of Gouy phase $\Psi_{0 l}(z)$ for $l=0,1$, and 2. The beam waist is located at $z=0$. As the measurement position $z$ moves from $-\infty$ to $\infty, \Psi_{0 l}(z)$ changes from $-\pi l / 2$ to $\pi l / 2$. Thus, the phase difference $\delta \Psi(z)$ between the two LG modes $l=0$ and $l=1$ changes from $-\pi / 2$ to $\pi / 2$. This means that the phase difference at a certain point between the two LG modes can be varied by moving the beam waist position, i.e., by scanning the position of the focusing lens. This phase control can be used between more than two LG modes.

Figure 2 shows the experimental setup to observe quantum correlation in LG modes using Gouy phase shift. The photon pairs are produced by SPDC using a $\beta$-barium borate (BBO) crystal. The pump light is focused by $L_{p}$; the beam waist is located in the plane of a thin BBO crystal with a beam radius of $\omega_{p}$. In the idler path, the relative phase of the measurement mode is manipulated by scanning the hologram $H_{I}$ two-dimensionally; by contrast, in the signal path, it is manipulated using the Gouy phase shift by varying the longitudinal position of $L_{S 1}$. We then analyzed the coincidence events between two photon counters $D_{I}$ and $D_{S}$.

In the following, we outline the derivation of coincidence count probabilities [27] taking the effect of Gouy phase into account. When the pump beam is in $\mathrm{LG}_{00}$ mode, the two-photon state at the plane of the thin quadratic BBO crystal can be written as $[25,28]$

$$
|\Phi\rangle=\sum_{l=-\infty}^{\infty} \sum_{p, p^{\prime}=0}^{\infty} C_{p p^{\prime}}^{l}\left|p-l\left(\omega_{0 I}, 0\right)\right\rangle_{S}\left|p^{\prime} l\left(\omega_{0 I}, 0\right)\right\rangle_{I}
$$

where $\omega_{0 I}$ is the beam radius of the set of $\mathrm{LG}_{p l}$ modes used for expansion. The amplitude $C_{p p^{\prime}}^{l}$ is determined by the

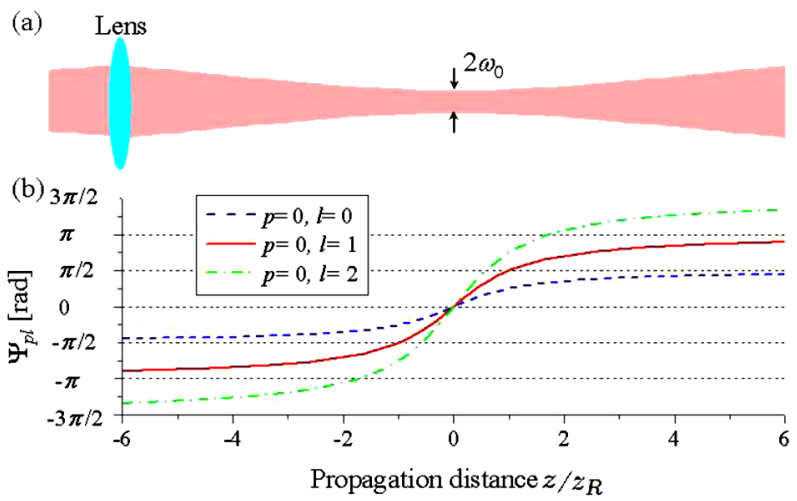

FIG. 1 (color online). (a) Variation of beam diameter with propagation distance $z$ for a LG beam focused by a lens; (b) Plot of $\Psi_{00}, \Psi_{01}$ and $\Psi_{02}$ against $z$. ratio of $\omega_{0 I}$ to $\omega_{p}$. Here, the direction of $z$ is the same as the propagation direction of the pump beam, and its origin $(z=0)$ is at the thin BBO crystal.

Although $\omega_{0 I}$ can be arbitrarily selected for calculation, we chose an appropriate value for $\omega_{0 I}$ in order to simplify the analysis. Since we use single mode fibers before the photon detector, only photons in a particular $\mathrm{LG}_{00}$ mode coupled to the fiber are counted. Thus, basically this $\mathrm{LG}_{00}$ mode back-propagated through the lenses and holograms to the BBO crystal can be considered as the measurement mode. In the idler path, the beam waist of this backpropagated mode is first positioned at the hologram $H_{I}$, and then the first-order diffraction beam is focused by the lens $L_{I 1}$ so that the waist of the second beam is located at the crystal. In the absence of phase modulation by the hologram, the radius of the waist of the second beam will be $\omega_{0 I}^{\prime}$. However, we have to carefully consider the effect of the hologram. When a $\mathrm{LG}_{00}$ mode is diffracted by $H_{I}$, a displaced phase singularity is added and the mode becomes a superposition of an infinite number of $\mathrm{LG}_{p l}$ modes [11]. Fortunately, however, it can be well approximated by a superposition of only $\mathrm{LG}_{00}$ and $\mathrm{LG}_{01}$ modes when the beam radius of the diffracted mode is chosen to be 0.8 times that of the incident mode, with the sum of the mode weight of $\mathrm{LG}_{00}$ and $\mathrm{LG}_{01}$ being no less than $85.9 \%$ [27]. Therefore, in the subsequent analysis, we choose $\omega_{0 I}=$ $0.8 \omega_{0 I}^{\prime}$. Under this approximation, the idler path's measurement mode at the plane of the BBO crystal can be written as

$$
\begin{aligned}
\left|I_{\text {basis }}\left(r_{I}, \theta_{I}\right)\right\rangle \propto & e^{-i \theta_{I}} \alpha_{I}\left(r_{I}\right)\left|00\left(\omega_{0 I}, 0\right)\right\rangle_{I} \\
& +\beta_{I}\left(r_{I}\right)\left|01\left(\omega_{0 I}, 0\right)\right\rangle_{I},
\end{aligned}
$$

where $\left(r_{I}, \theta_{I}\right)$ is the position of the dislocation of the hologram $H_{I}$ from the central axis of the back-propagated $\mathrm{LG}_{00}$ mode (inset of Fig. 2). The amplitude $\alpha_{I}\left(r_{I}\right)$ and $\beta_{I}\left(r_{I}\right)$ are real functions of $r_{I}$ which are either positive for all values of $r_{I}$ or always negative for all values of $r_{I}$. The relative phase between the two states depends only on $\theta_{I}$.

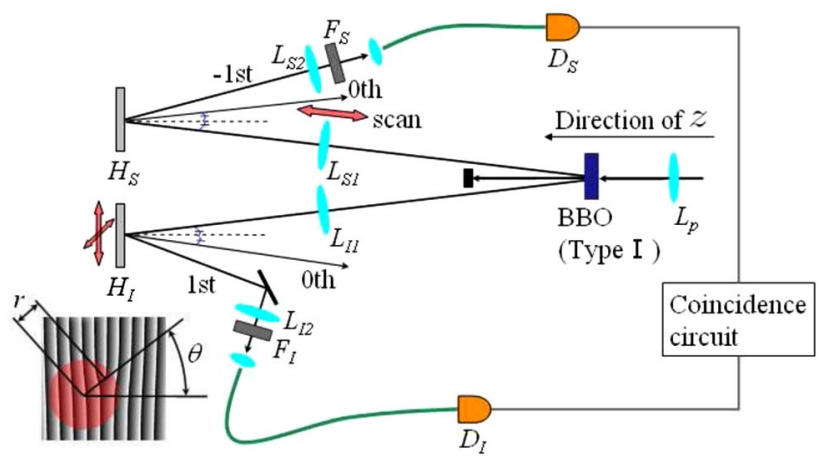

FIG. 2 (color online). The experimental setup for observing quantum correlation using Gouy phase shift. The inset shows the hologram pattern as viewed from the BBO crystal. 
When a photon is detected in the idler path, the projected mode in the signal path in the plane of the BBO crystal can be calculated as the inner product of Eqs. (3) and (4). For $\omega_{0 I} / \omega_{p}=0.35$, which is the condition used in our experiment, the amplitude when $p \geq 1$ is negligibly small [29]. By using this approximation, the projected mode in signal path can be written as

$$
\begin{aligned}
\left|S_{\text {state }}\left(r_{I}, \theta_{I}\right)\right\rangle \propto & e^{i \theta_{I}} C_{00}^{0} \alpha_{I}\left(r_{I}\right)\left|00\left(\omega_{0 I}, 0\right)\right\rangle_{S} \\
& +C_{00}^{1} \beta_{I}\left(r_{I}\right)\left|0-1\left(\omega_{0 I}, 0\right)\right\rangle_{S} .
\end{aligned}
$$

Similarly, measurement mode in the signal path reads

$$
\begin{aligned}
\left|S_{\text {basis }}\left(r_{S}, \theta_{S}\right)\right\rangle \propto & e^{i \theta_{S}} \alpha_{S}\left(r_{S}\right)\left|00\left(\omega_{0 S}, z_{0 S}\right)\right\rangle_{S} \\
& +\beta_{S}\left(r_{S}\right)\left|0-1\left(\omega_{0 S}, z_{0 S}\right)\right\rangle_{S},
\end{aligned}
$$

where $\left(r_{S}, \theta_{S}\right)$ is the position of the dislocation of $H_{S}$ from the central axis of the back propagated mode, $z_{0 S}$ is the beam waist position of the measurement mode, and $\omega_{0 S}$ is determined in the same way as $\omega_{0 I}$, i.e., $\omega_{0 S}=0.8 \omega_{0 S}^{\prime}$ where $\omega_{0 S}^{\prime}$ is the radius of the back-propagated mode in the absence of modulation by the hologram.

From Eqs. (5) and (6), and by executing four overlap integrations, we obtain an expression for the coincidence probability as follows:

$$
\begin{aligned}
P & =\mid\left\langle S_{\text {basis }}\left(r_{S}, \theta_{S}\right)\left|\left\langle I_{\text {basis }}\left(r_{I}, \theta_{I}\right) \mid \Phi\right\rangle\right|^{2}\right. \\
& \propto A+B \cos \left\{-\arctan \left(\frac{z_{0 S}}{z_{R I}+z_{R S}}\right)-\theta_{I}+\theta_{S}\right\},
\end{aligned}
$$

where $z_{R I}$ and $z_{R S}$ are, respectively, the Rayleigh range of the measurement mode in the idler and signal paths, and $A$ and $B$ are positive real numbers [30]. At $\theta_{I}^{\max }=\theta_{S}-$ $\arctan \left[z_{0 S} /\left(z_{R I}+z_{R S}\right)\right], P$ is a maximum, and at $\theta_{I}^{\min }=$ $\theta_{I}^{\max }+\pi, P$ is a minimum. Since arctan is a monotonic function, $\theta_{I}^{\max }$ and $\theta_{I}^{\min }$ change monotonically with $z_{0 S}$. This implies that the maximum and minimum positions rotate when $z_{O S}$ is scanned.

In the experiment (Fig. 1), a cw argon-ion laser (wavelength: $351 \mathrm{~nm}$; power: $60 \mathrm{~mW}$ ) was focused by the lens $L_{p}(f=900 \mathrm{~mm})$ to the beam radius $\omega_{p}=178 \mu \mathrm{m}$ in the BBO crystal (Type I, thickness: $3 \mathrm{~mm}$ ). The lenses $L_{S 1}$, $L_{I 1}, L_{S 2}$, and $L_{I 2}$ had $f=200 \mathrm{~mm}$. The distance from the BBO crystal to the hologram $H_{S}\left(H_{I}\right)$ was $1030 \mathrm{~mm}$. The holograms $(2 \mathrm{~mm} \phi$, pitch: $6.3 \mu \mathrm{m})$ were made by fabricating the structure on a thin polymer layer on a glass substrate using an electron beam writer, and then coating with gold [31]. $F_{I}$ and $F_{S}$ were narrow band-pass filters (702 nm, FWHM: $4 \mathrm{~nm}$ ). The photons were coupled to single mode fibers and detected by single photon detectors $D_{S}$ and $D_{I}$ (AQR-FC, Perkin Elmer). By changing the position of $L_{S 1}$, we measured the coincidence counting rates while scanning the position of $H_{I}$ two dimensionally [24]. The vertical and horizontal positions were shifted in steps of $7 \mu \mathrm{m}$ for a grid consisting of $21 \times 21$ points.
Figures $3(\mathrm{a})-3(\mathrm{~g})$ are coincidence count rate results obtained for seven different positions of lens $L_{S 1}$, shown as $2 \mathrm{D}$ maps of the position of hologram $H_{I}$. The position of hologram $H_{S}$ was fixed at $\left(r_{S}, \theta_{S}\right)=(27 \mu \mathrm{m}, 0)$. The count rates $(C)$ are normalized using the minimum and maximum count rates $\left(C^{\min }, C^{\max }\right)$ in each map [i.e., $\left.C_{\text {norm }}=\left(C-C^{\min }\right) /\left(C^{\max }-C^{\min }\right)\right]$. The typical minimum and maximum count rates are approximately 10 and 600 counts/s, respectively. The horizontal (vertical) axis denote the horizontal (vertical) position of $H_{I}$, in each $2 \mathrm{D}$ map. $z_{0 S}$ was determined experimentally using backpropagated light (reference light) with a wavelength $(680 \mathrm{~nm})$ close to that of the signal or idler photons (702 nm).

It can be clearly seen in Fig. 3 that the positions of maximum or minimum coincidences rotate clockwise around the origin as the position of the beam waist in signal path $\left(z_{0 S}\right)$ is scanned in the pump beam direction. The azimuthal coordinate of the maximum (minimum) in the 2D map indicates the phase of the two measurement basis states in the idler mode given by Eq. (4) where the maximum (minimum) coincidence events occurred. This result shows that the relative phase of the $\mathrm{LG}_{00}$ and $\mathrm{LG}_{0-1}$ measurement basis states in the signal path changed as the beam waist $z_{0 S}$ was displaced. This phase is not explicit in Eq. (6), but, as was previously discussed for Eq. (2), enters through the Gouy phase shift $\Psi_{p l}$ in Eq. (1). Because of the quantum entanglement in Eq. (3), the phase changed on rotation of the maximum or minimum position. The continuity of the change also proves the quantum coherence in the correlation shown in Eq. (3).

To analyze the data in Fig. 3, we introduce parameter $\theta_{\text {map }}$ which is the angle of a line segment between the maximum and minimum coincidence points to the horizontal axis (see Fig. 3). Figure 4 is a plot of $\theta_{\text {map }}$ (square) against $z_{0 S}$.
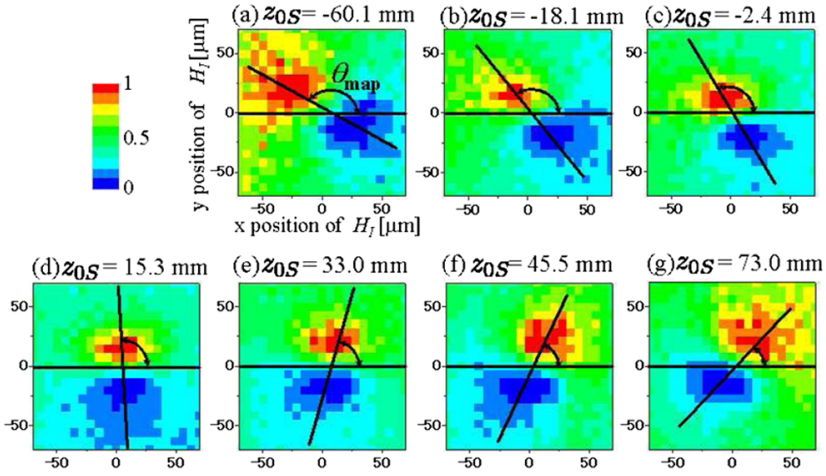

FIG. 3 (color online). Maps of the normalized coincidence count rates obtained by scanning hologram $H_{I}$. The distances between $H_{S}$ and $L_{S 1}$ were (a) $250 \mathrm{~mm}$, (b) $255 \mathrm{~mm}$, (c) $257.5 \mathrm{~mm}$, (d) $260 \mathrm{~mm}$, (e) $262.5 \mathrm{~mm}$, (f) $265 \mathrm{~mm}$, and (g) $270 \mathrm{~mm}$. 


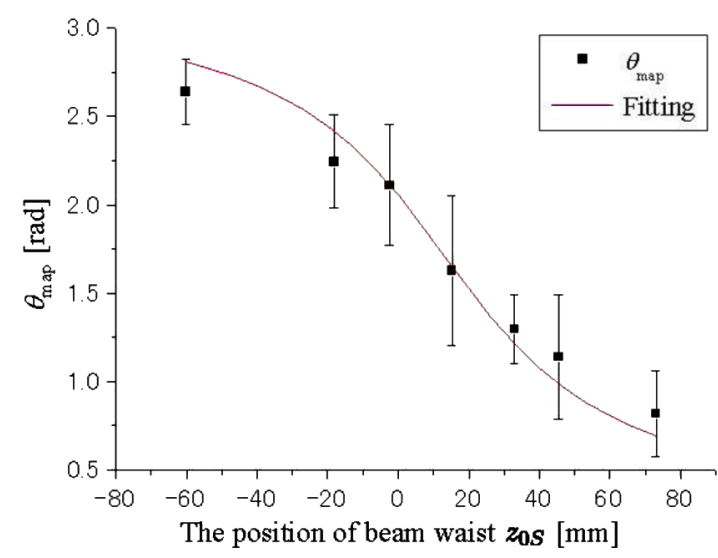

FIG. 4 (color online). Dependence of $\theta_{\text {map }}$ on the position of beam waist $z_{0 S}$. The squares represent the measured data and the solid line represents the fitting result. The error bars are determined based on the assumption that the minimum (maximum) points exist in the areas $6.25 \%$ of the largest (smallest) coincidence counts in Fig. 3.

Noting that $\theta_{I}^{\max }=\theta_{S}-\arctan \left[z_{0 S} /\left(z_{R I}+z_{R S}\right)\right]$ and $\theta_{I}^{\min }=\theta_{I}^{\max }+\pi$ obtained from Eq. (7), $\theta_{\text {map }}$ must be $\theta_{I}^{\max }$ theoretically. From this, we define the fitting function for $\theta_{\text {map }}$ as follows:

$$
\theta_{\text {map }}^{\text {fit }}=-\arctan \left(\frac{z_{0 S}-z_{\text {offset }}}{z_{R I}+z_{R S}}\right)+\theta_{\text {offset }},
$$

where $z_{\text {offset }}$ and $\theta_{\text {offset }}$ are the fitting parameters denoting the offset of the beam waist position of the measurement mode in the BBO crystal and in the holograms $H_{I}$ (or $\left.H_{S}\right)$. The solid line in Fig. 3 is the fitting result for $\theta_{\text {map. The }}$ Rayleigh ranges were determined by using $\omega_{0 I, S}=$ $0.8 \omega_{0 I, S}^{\prime}$, where measured beam radiuses of reference light $\omega_{0 I}^{\prime}=78 \mu \mathrm{m}$ and $\omega_{0 S}^{\prime}=70 \sim 96 \mu \mathrm{m}$ [32]. The gradient of the fitting curve reproduces the experimental data well.

When a hologram is shifted and scanned to change the phase between LG modes, the original superposition state is not maintained but converted into the Gaussian mode. On the other hand, our proposed method using Gouy phase shift only changes the relative phase while preserving the relative amplitudes of the original superposition states. In this sense, our method provides a nondestructive method to control relative phases for multidimensional photonic states. It is also possible to extend our scheme for quantum tomography and Bell inequality test using the superposition state of $\mathrm{LG}_{00}$ and $\mathrm{LG}_{02}$ modes.

We thank J. Hamazaki, R. Morita, and R. Okamoto for helpful discussion. This work was supported by the Japan Science and Technology Agency (JST), Ministry of Internal Affairs and Communication (MIC), Japan Society for the Promotion of Science (JSPS), 21st Century COE Program, Special Coordination Funds for
Promoting Science and Technology.

*takeuchi@es.hokudai.ac.jp

[1] A. Ekert, Phys. Rev. Lett. 67, 661 (1991).

[2] P. Walther et al., Nature (London) 434, 169 (2005).

[3] C. H. Bennett and S. J. Wiesner, Phys. Rev. Lett. 69, 2881 (1992).

[4] C. H. Bennett et al., Phys. Rev. Lett. 70, 1895 (1993).

[5] D. Kaszlikowski et al., Phys. Rev. Lett. 85, 4418 (2000).

[6] N. J. Cerf, M. Bourennane, A. Karlsson, and N. Gisin, Phys. Rev. Lett. 88, 127902 (2002).

[7] H. Nihira and C. R. Stroud, Jr., Phys. Rev. A 72, 022337 (2005).

[8] A. Mair, A. Vaziri, G. Weihs, and A. Zeilinger, Nature (London) 412, 313 (2001).

[9] A. Vaziri, G. Weihs, and A. Zeilinger, Phys. Rev. Lett. 89, 240401 (2002).

[10] N. K. Langford et al., Phys. Rev. Lett. 93, 053601 (2004).

[11] G. Molina-Terriza, A. Vaziri, J. Řeháček, Z. Hradil, and A. Zeilinger, Phys. Rev. Lett. 92, 167903 (2004).

[12] J. T. Barreiro et al., Phys. Rev. Lett. 95, 260501 (2005).

[13] H. He, M.E. Friese, N.R. Heckenberg, and H. Rubinsztein-Dunlop, Phys. Rev. Lett. 75, 826 (1995).

[14] L. Allen, M. W. Beijersbergen, R. J. C. Spreeuw, and J. P. Woerdman, Phys. Rev. A 45, 8185 (1992).

[15] C. R. Gouy, C. R. Acad. Sci. Paris Ser. IV 110, 1251 (1890).

[16] C. R. Gouy, Ann. Chim. Phys. Ser. 6, 24, 145 (1891).

[17] Simin. Feng, and H. Winful, Opt. Lett. 26, 485 (2001).

[18] M. V. Berry, Proc. R. Soc. A 392, 45 (1984).

[19] A. B. Ruffin et al., Phys. Rev. Lett. 83, 3410 (1999).

[20] R. W. McGown, R. A. Cheville, and D. Grischkowsky, Appl. Phys. Lett. 76, 670 (2000).

[21] F. Lindner et al., Phys. Rev. Lett. 92, 113001 (2004).

[22] J. Hamazaki, Y. Mineta, K. Oka, and R. Morita, Opt. Express 14, 8382 (2006).

[23] J. H. Chow, G. de Vine, M. B. Gray, and D. E. McClelland, Opt. Lett. 29, 2339 (2004).

[24] D. Kawase, S. Takeuchi, K. Sasaki, A. Wada, Y. Miyamoto, and M. Takeda, arXiv:quant-ph/0602199v1.

[25] Juan P. Torres, Yana Deyanova, Lluis Torner, and G. Molina-Terriza, Phys. Rev. A 67, 052313 (2003).

[26] S. Franke-Arnold, S. M. Barnett, M. J. Padgett, and L. Allen, Phys. Rev. A 65, 033823 (2002).

[27] D. Kawase, Y. Miyamoto, M. Takeda, K. Sasaki, and S. Takeuchi (to be published).

[28] J.P. Torres, A. Alexandrescu, and Lluis Torner, Phys. Rev. A 68, 050301 (2003).

[29] The total amount of these components is less than $0.5 \%$.

[30] Note that the denominator in the arctan in Eq. (7) is not $z_{R S}$ but $z_{R I}+z_{R S}$. This is due to the entanglement of mode properties (curvature and beam radius) in the signal and the idler paths.

[31] Y. Miyamoto, M. Masuda, A. Wada, and M. Takeda, in Proc. SPIE 3740, 232 (1999).

[32] $\omega_{0 S}^{\prime}\left(z_{0 S}\right)$ are determined experimentally using the reference light, and $z_{R S}$ is derived using $z_{R}=k \omega_{0}^{2} / 2$ with $\omega_{0 S}=0.8 \omega_{0 S}^{\prime}$. 